\section{The effect of visual and tactual stimulation on learning of abstract forms*}

\author{
JAMES M. FICO \\ University of North Dakota, Grand Forks, N. Dak. 58201 \\ and \\ HOWARD S. BRODSKY \\ University of Wisconsin-Milwaukee, Milwaukee, Wis. 53201
}

Four experiments were performed to explore visual, tactual, and combined visual-tactual learning in adults. A recognition experiment demonstrated visual training or testing to be superior to tactual training or testing. A recal experiment showed visual learning to be superior to both tactual learning and combined visual-tactual learning. A pretraining procedure, using either familiar or unfamiliar tactual stimuli, showed that a lack of experience with tactual stimuli did not affect performance. In an experiment varying order of presentation, Ss receiving visual stimulation before tactual stimulation recalled more than Ss receiving the reverse order. The results were interpreted as indicating the superiority of visual information processing. Combining tactual with visual stimulation resulted in decreased performance.

The investigation of visual vs tactual learning of abstract forms has produced conflicting results. In a study of cross-modality transfer, Gaydos (1956) had Ss first associate names with form stimuli until a criterion was reached and then learn shapes either visually or tactually, with relearning on the cross modality. Transfer-task performance was superior for those Ss presented with tactual stimulation first, followed by visual learning.

Lobb (1965), however, using a similar learning-relearning paradigm, had eighth-grade students identify a standard stimulus in a form recognition task. Visual or tactual learning was followed either by visual or tactual testing. Lobb found visual training superior to tactual training for unimodal tasks. The crossmodal groups performed intermediate to the unimodal groups.

In two replications, Cashdan (1968) reported inconsistent results in cross-modality visual and tactual learning. Cashdan suggested that tactual stimuli are perceived as a successive stimulation process, due to S's inability to perce:ve the entire stimulus at one time. Visual stimulation is, by comparison, relatively simultaneous in that the entire stimulus can be perceived at once. To control for this difference, Cashdan allowed Ss to learn Gaydos's stimuli either visually or tactually through successive stimulation. Lobb's findings were supported in the first experiment, with a visual exposure and tactual test resulting in significantly

* Requests for reprints should be sent to James M. Fico, Department of Psychology, University of North Dakota, Grand Forks, N. Dak. 58201 . greater performance. In a second replication, however, the reverse

In a more recent study of bimodal learning, Cashdan \& Zung (1970) found that visual input, when supplemented with simultaneous tactual input, resulted in similar performance levels, as did exclusively visual input.

investigated visual (V) and tactual (T) learning of abstract shapes. The simultaneous presentation of visual and tactual (VT) stimulation was compared with the unimodal experiences. Also, comparisons were made of recognition and recall testing as well as the effect of pretraining experience. A recall test procedure, introduced by Rock \& Victor (1964) was incorporated into later experiments because of its apparent increased sensitivity in relation to recognition testing. The effect of tactual pretraining experience was investigated in an effort to clarify Cashdan's (1968) result involving the absence of an effect on performance due to tactual practice. Also, crossmodal transfer was studied through a comparison of presentation order because of the above-mentioned inconsistency of previous findings.

\section{EXPERIMENT 1 \\ Subjects}

In all four experiments, Ss were students enrolled in introductory psychology courses who received course credit for serving as Ss. Each group comprised $12 \mathrm{Ss}$, and each $\mathrm{S}$ was tested individually.

\section{Apparatus}

The shapes used for both visual and iactual stimulation were cut from 3/8-in. white styrofoam, measuring $4 \frac{1}{2}$ in. at their largest dimension. a maximum of 13 points. Color slides finding was reported. These shapes had a minimum of 5 and of the stimuli were used as visual stimuli. The shapes were projected at actual size. Tactual stimuli were mounted on $9 \times 10 \mathrm{in}$. Masonite boards to provide a uniform background. These boards were inserted into an immobile frame to assure consistent orientation. Separate sets of tactual stimuli were used during training and testing. A black box with an open area through which the Masonite boards were placed was used for the uniform presentation of stimuli. Tactual stimuli were not visible to $\mathrm{Ss}$.

\section{Procedure}

The $108 \mathrm{Ss}$ were assigned randomly to nine recognition groups on the basis of modality of training $(\mathrm{V}, \mathrm{VT}, \mathrm{T})$ and modality of testing (V, VT, T). Each S was instructed that he would see, feel, or see and feel 20 shapes. No information was given $S$ regarding subsequent testing. These shapes were presented for $15 \mathrm{sec}$ and were followed by a 15 -sec intertrial interval. Each shape was presented only once. No practice stimuli were presented. Visual stimuli were experienced by looking at the screen. Tactual stimuli were experienced by feeling beneath a panel which blocked S's view of the tactual stimulus. Visual-tactual stimuli were experienced by simultaneously looking at the screen and feeling the stimuli below the screen. Recognition testing began immediately after training. Ss were instructed to say "yes" if they recognized or "no" if they did not recognize the 40 test stimuli. Twenty of these test stimuli were the original training stimuli. The distractor items were similar in nature but different in detail.

\section{Results}

Totals for correct positive ("yes") responses expressed as proportions of the total possible number correct are shown in Table 1. Chance performance for each $\mathbf{S}$ was 10 positive recognitions and 120 positive recognitions for each group of 12 Ss. Only the tactual training and tactual testing $S$ performed below this level.

An analysis of variance (Training by Testing) revealed a significant effect for the training modality, $F(2,99)=$ $10.38, \mathrm{p}<.01$, and the testing modality, $F(2,99)=19.53, p<.01$. The interaction between training and testing modalities was not significant, $\mathbf{F}<1$. A Newman-Keuls analysis showed visual training to be superior to tactual training and visual testing to be superior to tactual testing, $\mathrm{p}<.05$ in each case. On either training or testing, combined visual-tactual experience did not differ from unimodal visual or tactual experience.

\section{EXPERIMENT 2}

$$
\text { Method }
$$

Thirty-six Ss were assigned randomly to three recall groups on the 
Table 1

Proportion of Total Correct Positive Recognition Scores With 12 Ss Per Cell Testing

\begin{tabular}{lllll} 
Training & $\mathrm{V}$ & $\mathrm{VT}$ & $\mathrm{T}$ & Total \\
\hline V & .758 & .770 & .550 & .693 \\
VT & .733 & .712 & .508 & .651 \\
T & .595 & .575 & .441 & .537 \\
Total & .695 & .686 & .500 & .627
\end{tabular}

Note-Chance performance was a proportion of .500 positive recognition for each cell of $12 \mathrm{Ss}$.

basis of modality of learning ( $\mathrm{V}, \mathrm{VT}$, $\mathrm{T})$. Training procedures were identical to those in Experiment 1 . The same shapes were employed. The Ss were tested by drawing as many of the shapes as they could during a 10-min period. The shapes were scored by a grader who was blind with respect to the design of the $E$. A rigid set of criteria was employed to ensure reliability. A correct response necessitated complete recall of the entire stimulus. Partial results were also scored but not reported due to lack of differentiation between groups. The drawings were also scored by $\mathrm{E}$, and $81 \%$ of these scores were identical to the grader's scores and $94 \%$ were within 1 point.

To indicate a chance level of performance on the recall test, $12 \mathrm{Ss}$ served as a control group who did not receive any training.

Results and Discussion

Tactually trained Ss correctly drew 21 shapes, V-T Ss drew 57 shapes, and V Ss drew 83 shapes. Control Ss drew no shapes which were judged as correct. A Newman-Keuls test yielded significant differences $(p<.05)$ between each of the training groups. Tactually trained Ss drew a larger number of correct shapes than the no-training control group, $\mathrm{p}<.05$, indicating that some learning did occur for this group.

The recall $S$ s were thus ordered (by decreasing performance) as $\mathrm{V}, \mathrm{VT}$, and $T$. This same order had resulted from recognition testing; however, the differences did not statistically differentiate the VT groups from the $\mathrm{V}$ or $\mathrm{T}$ groups. The recall test was thus more sensitive to the differences in performance of these groups, and for that reason, recall testing was used exclusively in following experimentation.

The lower performance of VT Ss compared to V Ss might have been due to the novelty of the tactual experience. To eliminate this possibility, Experiment 3 was conducted to test the effect of tactual pretraining experience on this difference.

\section{EXPERIMENT 3}

\section{Method}

The 108 Ss were assigned randomly to nine recall groups on the basis of modality of training and type of tactual pretraining. Tactual pretraining experience was either with familiar stimuli (F), unfamiliar stimuli (U), or no pretraining. Stimuli $F$ were single English letters or Arabic numerals. To ensure that Ss correctly identified F stimuli, they were asked to identify the stimuli as they were experienced. Stimuli $U$ were similar in difficulty to the experimental shapes and had been used in Experiment 1 as the incorrect recognition choices.

Each pretraining stimulus was exposed for $15 \mathrm{sec}$. Ss were then immediately introduced to their respective experimental training condition. Each pretraining procedure was employed across $\mathrm{V}, \mathrm{T}$, and VT training according to the same training and teng procedures as Experiment 2. Again, the same shapes were used for training stimuli.

\section{Results and Discussion}

The number of shapes correctly drawn, again expressed as a proportion, for each group is presented in Table 2. An analysis of variance (Modality of Training by Type of Pretraining) showed no effect of the pretraining variable, $F(2,99)=$ .01 . The effect of modality of training was significant, $F(2,99)=58.32$, $\mathrm{p}<.01$. As in Experiment 2, the best performance was made by the $V$ group, followed by the VT and $T$ groups. Group VT drew fewer correct shapes than Group V, and drew more correct shapes than Group $T, p<.05$ in each case. Experiment 3 then completely replicated the findings of Experiment 2.

The finding that $S$ s wo receive relevant tactual experience simultaneously with visual stimulation recall less than $S s$ who receive that visual stimulation alone suggests that the decrement is due to an effor by $S s$ to integrate information from the tactual experience. Experiment 4 served the purpose of separating temporally the visual and tactual components of visual-tactual learning. Thus, two orders of presentation are indicated, V-T and T-V.

\section{EXPERIMENT 4 \\ Method}

Two groups of $12 \mathrm{Ss}$ received $30 \mathrm{sec}$ of cross-modality training on each stimulus. Group V-T received the 20 training stimuli in the order of $15 \mathrm{sec}$ of visual training, followed by $15 \mathrm{sec}$ of tactual experience with the same stimulus. Group T.V experienced the tactual stimulus for $15 \mathrm{sec}$ followed by visual experience for $15 \mathrm{sec}$. To equate total training time with previous groups, the 15-sec ISI was eliminated; Ss received a total of $10 \mathrm{~min}$ of stimulus exposure. Recall testing was administered as in Experiments 2 and 3 .

Results and Discussion

Group V-T Ss correctly recalled a total of 71 stimuli, while Group T-V Ss recalled 37 stimuli. The difference was found to be significant $[\mathrm{t}(22)=$ $3.90, \mathrm{p}<.01\}$.

In the case of V-T learning, $S$ s had the opportunity to learn the shapes identically to the $\mathrm{V}$ training procedure in the previous experiments, with the modification that the previous interstimulus interval was replaced by a tactual experience on the same stimulus. Group T-V differed from Group V-T only in order of modality experience. However, the tactual first exposure to the stimulus seemingly heightened S's effort to integrate the tactual stimulation into their perception of the stimulus.

\section{CONCLUSIONS}

Using a recognition test, the visual modality yielded performance superior to the tactual mode in training and testing. Using a recall testing procedure, visual training was again superior to tactual training; however, the combined visual and tactual training was between and significantly different from the other two groups.

When two types of pretraining were used, modality of training again resulted in the $\mathrm{V}, \mathrm{VT}, \mathrm{T}$ ordering of groups, thus replicating the findings of Experiment 2.

Pretraining of the type used in

Table 2

Proportion of Number of Correct Recall Responses for Ss Following Pretraining

\begin{tabular}{lcccc} 
& No Pretraining & Familiar & Unfamiliar & Total \\
\hline V & .312 & .312 & .354 & .326 \\
VT & .254 & .225 & .237 & .238 \\
T & .054 & .095 & .037 & .062 \\
Total & .206 & .211 & .209 & .209 \\
\hline
\end{tabular}

Note-Chance performance for recall groups was .000 proportion of correct responses, as indicated by an unreported group of $12 \mathrm{Ss}$ who received no training. 
Table 3

Summary of Recall Groups Without Pretraining

\begin{tabular}{lccc}
\hline & \multicolumn{2}{c}{ Training Procedure } \\
\cline { 2 - 4 } Group & $0-15$ Sec & 15-30 Sec & Total \\
\hline T & T & - & .054 \\
T-V & T & V & .154 \\
VT & V and T & - & .254 \\
V-T & V & T & .295 \\
V & V & - & .312 \\
\hline
\end{tabular}

Note Chance performance for recall groups, again, was .000 proportion of correct responses.

Experiment 3 showed no systematic effect on recall performance.

In Experiment 4, the order of presentation of stimulus modality was found to be significant. The Ss who visually experienced the stimulus prior to experience with it tactually recalled more stimuli than Ss who tactually experienced the stimuli before visually experiencing them.

The present research clarifies Cashdan's (1968) question of presentation order and supports
Lobb's (1965) earlier findings. The visual-tactual temporal sequence of presentation was here found to be superior to the tactual-visual order, as was indicated in Cashdan's Replication 1. However, the present authors cannot concur with a later interpretation (Cashdan \& Zung, 1970 ) that relegates the tactual experience that follows visual stimulation to the role of redundancy. While Cashdan and Zung's visual and haptic groups performed both above and below the levels of their visual Ss, all four experiments described here showed inferior performance in either order of crossmodal training, whether presented simultaneously or sequentially (see Tables 1,2 , and 3 ). Thus, the tactual stimulation that follows visual learning (Group VT) must be treated as distraction rather than as a learning experience. This hypothesis is suggested by the no-pretraining recall groups, $\mathrm{V}$ and $\mathrm{T}$. Though the visual training followed by delay yielded greater performance than did the tactual experience, the difference in magnitude fails to indicate empirical support.

Finally, in future related investigations, the recall procedure employed here seems preferred over the recognition method because it eliminates the interpretative difficulties occasioned by the presence of substantial levels of performance attributable merely to chance when the latter method is employed.

\section{REFERENCES}

CASHDAN, S. Visual and haptic form discrimination under conditions of successive stimulation. Journal of Experimental Psychology, 1968, 76, 215-218.

CASHDAN, S., \& ZUNG, B. J. Effect ot sensory modality and delay on form recognition. Journal of Experimental Psychology, 1970,86, 458-460.

GAYDOS, H. F. Intersensory transfer in the discrimination of form. American Journal of Psychology, 1956, 69, 107-110.

LOBB, H. Vision versus touch in form discrimination. Canadian Journal of Psy chology, 1965, 19, 175-187.

ROCK, I \& VICTOR J Vision and touch: An experimentally created conflict between the two senses. Science, 1964, 143, 594-596. 